TRANSACTIONS OF THE

AMERICAN MATHEMATICAL SOCIETY

Volume 186. December 1973

\title{
EXTENDING CELL-LIKE MAPS ON MANIFOLDS
}

BY

\author{
B. J. BALL AND R. B. SHER( 1 )
}

ABSTRACT. Let $X$ be a closed subset of a manifold $M$ and $G_{0}$ be a celllike upper semicontinuous decomposition of $X$. We consider the problem of extending $G_{0}$ to a cell-like upper semicontinuous decomposition $G$ of $M$ such that $M / G \approx M$. Under fairly weak restrictions (which vanish if $M=E^{n}$ or $S^{n}$ and $n \neq 4$ ) we show that such a $G$ exists if and only if the trivial extension of $G_{0}$, obtained by adjoining to $G_{0}$ the singletons of $M-X$, has the desired property. In particular, the nondegenerate elements of Bing's dogbone decomposition of $E^{3}$ are not elements of any cell-like upper semicontinuous decomposition $G$ of $E^{3}$ such that $E^{3} / G \approx E^{3}$. Call a cell-like upper semicontinuous decomposition $G$ of a metric space $X$ simple if $X / G \approx X$ and say that the closed set $Y$ is simply embedded in $X$ if each simple decomposition of $Y$ extends trivially to a simple decomposition of $X$. We show that tame manifolds in $E^{3}$ are simply embedded and, with some additional restrictions, obtain a similar result for a locally flat $k$-manifold in an m-manifold $(k, m \neq 4)$. Examples are given of an everywhere wild simply embedded simple closed curve in $E^{3}$ and of a compact absolute retract which embeds in $E^{3}$ yet has no simple embedding in $E^{3}$.

1. Introduction. We will be concerned with variations of the following general problem: If $X$ is a closed subset of a manifold $M$ and $f: X \rightarrow Y$ is a proper cell-like map of $X$ onto a metric space $Y$, under what conditions is it possible to extend $f$ to a proper cell-like map $F$ defined on all of $M$ such that $F(M)$ is homeomorphic to $M$ ? (We do not assume that the boundary of a manifold is necessarily empty.) For 3-manifolds, analogous questions have been considered by R. H. Bing [9] and others (see, for example, [G, [12], [18], [19] and the discussion in $\$ 15$ of [20]).

Since proper maps and upper semicontinuous decompositions of metric spaces correspond in a natural way, our results have equivalent formulations in terms of mappings and in terms of decompositions. In the latter terminology, the basic question considered becomes: Given a cell-like decomposition $G_{0}$ of $X$, when

Received by the editors March 3, 1972 and, in revised form, April 5, 1973. $57 \mathrm{~A} 60$.

AMS (MOS) subject classifications (1970). Primary 54C20; Secondary 57 A10, 57A15,

Key words and phrases. Extension, proper map, cell-like space, cell-like map, monotone map, trivial extension, upper semicontinuous decomposition, dogbone space, tame, locally flat.

(1) The second named author supported in part by NSF Grant GP-29585. 
is there a cell-like decomposition $G$ of $M$ such that $G_{0} \subset G$ and $M / G \approx M$ ? Under fairly weak restrictions (which vanish if $M$ is $S^{n}$ or $E^{n}$ ), we show that if $M$ is an $n$-manifold, $n \neq 4$, then such a $G$ exists only in case the trivial extension of $G_{0}$, obtained by adjoining to $G_{0}$ all singletons in $M-X$, has the desired property. In particular, the set of nondegenerate elements of Bing's dogbone decomposition of $E^{3}$ cannot be embedded in any cellular decomposition of $E^{3}$ which yields $E^{3}$ as its decomposition space, which provides a negative answer to a question raised by Bing in [9].

We say that a cell-like upper semicontinuous decomposition $G$ of a metric space $X$ is simple if $X / G \approx X$ and that a closed subset $Y$ of $X$ is simply embedded in $X$ if every simple decomposition of $Y$ can be trivially extended to a simple decomposition of $X$ (equivalently, if every proper cell-like map on $Y$ can be extended to a proper cell-like map $F$ on $X$ such that $F(X) \approx X$ and $F \mid X-Y$ is a homeomorphism onto $F(X)-F(Y)$ ). We show that every tame manifold in $E^{3}$ is simply embedded in $E^{3}$, and obtain similar results, with additional restrictions, for a locally flat $k$-manifold in an $m$-manifold ( $k, m \neq 4$ ).

Local flatness is by no means a necessary condition for a manifold in $E^{n}$ to be simply embedded; we show, in fact, that an arc in $E^{3}$ may be simply embedded even though it is wild at each of its points. With respect to more complicated sets, we observe that there exist 1-dimensional continua for which every embedding in $E^{3}$ is simple and, at the other extreme, show that a continuum may be embeddable in $E^{3}$ and yet have no simple embedding in $E^{3}$. It will be shown in a later paper that every circularly chainable continuum can be simply embedded in $E^{3}$, and the question of which continua have both simple and nonsimple embeddings in $E^{3}$ will be considered in greater detail.

2. Definitions and notation. Our usage of upper semicontinuous (collection or decomposition) and associated terms is standard (e.g., as in [30] and [2]).

If $X$ and $Y$ are metric spaces, a map $f: X \rightarrow Y$ is said to be closed if $f(C)$ is closed in $Y$ for every closed subset $C$ of $X$, and to be compact if $f^{-1}(K)$ is compact for every compact subset $K$ of $Y$. If $f$ is closed and $f^{-1}(y)$ is compact for each $y \in Y$, then $f$ is said to be proper; it readily follows that $f$ is proper if and only if it is compact.

We use the notation $f: X \rightarrow Y$ to indicate that $f$ maps $X$ onto $Y$ (of course $f: X \rightarrow Y$ does not preclude $f: X \rightarrow Y$ ).

It is well known that if $X$ and $Y$ are metric spaces and $f: X \rightarrow Y$ is a proper map, then the set $G=\left\{f^{-1}(y) \mid y \in Y\right\}$ is an upper semicontinuous decomposition of $X$ and $X / G \approx Y$; conversely, if $G$ is any upper semicontinuous 
decomposition of $X$, then $X / G$ is a metric space and the projection map $P$ : $X \rightarrow X / G$ is proper. Most of our results may be stated, equivalently, in terms of mappings and in terms of decompositions; generally, we give only one version formally, but may later use the alternative formulation without explicit comment.

By an n-manifold we mean a separable metric space each point of which has a neighborhood homeomorphic to a closed $n$-cell. As usual, if $M$ is a manifold, the set of all points of $M$ which have an open $n$-cell neighborhood in $M$ is called the interior of $M$ and is denoted by Int $M$; the set $M$ - Int $M$ is called the boundary of $M$, and will be denoted by $\partial M$. We do not assume that manifolds are compact or connected, but whenever we speak of a manifold $M$ as being "in" a manifold $N$, we always as sume that $M$ is embedded as a closed subset of $N$.

A subset $X$ of an $n$-manifold $M$ is said to be cellular in $M$ if $X$ is the intersection of a sequence $\left\{C_{i}\right\}$ of closed n-cells in $M$, with $C_{i+1} \subset$ Int $C_{i}$ for each $i$. A continuum which is homeomorphic to a cellular subset of some Euclidean space $E^{n}$ is said to be cell-like. A decomposition $G$ is said to be cell-like (cellular) if each element of $G$ is cell-like (cellular), and a mapping $f: X \rightarrow Y$ is cell-like if $f^{-1}(y)$ is cell-like for each $y \in Y$. (See [17] or [26].)

If $U$ is a subset of a space $X$, then $\mathrm{Cl} U$ will denote the closure of $U$ in $x$.

If $G$ is a collection of subsets of $X$, we use $G^{*}$ to denote the union of the elements of $G$, and if $C \subset X$, we let $G_{C}$ denote the set of all elements of $G$ which intersect $C$. A subset $C$ of $X$ is said to be saturated with respect to $G$ if $C=G_{C}^{*}$. If $f: X \rightarrow Y$, then $f(G)$ will denote $\{f(g) \mid g \in G\}$.

If $Y$ is a closed subset of a metric space $X$ and $G$ is an upper semicontinuous decomposition of $Y$, then any upper semicontinuous decomposition $G^{\prime}$ of $X$ such that $G \subset G^{\prime}$ will be called an extension of $G$. The extension of $G$ obtained by adding to $G$ all singleton subsets of $X-Y$ will be called the trivial extension of $G$ and will be said to be generated by $G$. This decomposition of $X$ will be denoted by $\tilde{G}(X)$, or simply $\widetilde{G}$ in case confusion is unlikely.

A cell-like upper semicontinuous decomposition $G$ of a metric space $X$ will be said to be simple if $X / G \approx X$ and to be locally simple if, for each $g \in G$, every neighborhood $U$ of $g$ in $X$ contains a closed neighborhood $C$ of $g$ in $X$ such that $G_{C}$ generates a simple decomposition of $X$. (In general, a locally simple decomposition of a metric space $X$ need not be simple, nor need a simple decomposition be locally simple. If $X$ is a manifold, however, the situation is markedly different, as shown below.) A closed subset $Y$ of $X$ is said to be simply embedded in $X$ if every simple decomposition of $Y$ generates a simple decomposition of $X$. 
3. Modifying cell-like maps on manifolds. The results of this section depend heavily on Siebenmann's recent theorem [26] on homeomorphic approximations of cell-like maps between $m$-manifolds (for $m=3$, this result was proved earlier by Armentrout ([2], [3], [4], [5])). Siebenmann's theorem includes some restrictions which we find it convenient to abbreviate as follows.

Conditions $S(M, f)$ and $S(M, G)$. If $M$ is an $m$-man ifold and $f: M \rightarrow Y$ is a cell-like map of $M$ onto a metric space $Y$, we will say that condition $S(M, f)$ holds provided that $m<3$ or

(1) if $m=3$, then for each $y \in Y, f^{-1}(y)$ has a neighborhood in $M$ which is embeddable in $E^{3}$,

(2) if $m=5$, then $f \mid \partial M$ is bijective,

(3) if $m=4$ or $m>5$, then for each $y \in Y, f^{-1}(y) \cap \partial M$ is either empty or cell-like.

If $G$ is an arbitrary collection of cell-like subsets of $M$, then $S(M, G)$ is defined analogously (i.e., by replacing $f^{-1}(y)$ in (1) and (3) by an arbitrary $g \in$ $G$ and requiring in (2) that no nondegenerate element of $G$ intersect $\partial M$ ).

The following lemma provides a slight additional simplification of the statement of Siebenmann's theorem. That the first part of this lemma holds was indicated in $\$ 3$ of [26], where it is also shown that the second part fails for $m \geq 4$.

3.1. Lemma. If $M$ and $N$ are m-manifolds and $f: M \rightarrow N$ is a proper cell-like map, then $f(\partial M)=\partial N$ and, if $m \leq 3, f \mid \partial M: \partial M \rightarrow \partial N$ is cell-like.

Proof. Suppose $x \in \partial M$. If $f(x) \in \operatorname{Int} N$, there is an open subset $U$ of $N$ such that $f(x) \in U$ and $U \approx E^{m}$. Then $f^{-1}(U)$ is an $m$-manifold with nonempty boundary, and since $f \mid f^{-1}(U): f^{-1}(U) \rightarrow U$ is a proper homotopy equivalence [17], it follows that $H_{0}\left(f^{-1}(U), \partial f^{-1}(U)\right) \approx H_{C}^{m}\left(f^{-1}(U)\right) \approx H_{C}^{m}(U) \approx H_{C}^{m}\left(E^{m}\right)=Z$. (Here $H_{i}$ denotes homology with integral coefficients and $H_{C}^{i}$ cohomology with integral coefficients and compact supports.) But this is a contradiction since $f^{-1}(U)$ is path connected and $\partial f^{-1}(U) \neq \varnothing$. It follows that $f(\partial M) \subset \partial N$; a similar argument shows that if $y \in \partial N$, then $f^{-1}(y) \cap \partial M \neq \varnothing$, and hence $f(\partial M)=\partial N$.

If $m<3$, it is easy to show directly that $f \mid \partial M$ is monotone and therefore cell-like, so suppose $m=3$. If $y \in \partial N$ and $W$ is an open subset of $\partial M$ containing $f^{-1}(y) \cap \partial M$, there is an open subset $V$ of $N$ containing $y$ such that $V \approx E_{+}^{3}$ and $f^{-1}(V) \cap \partial M C W$. Then, as above, $H_{2}\left(f^{-1}(V), \partial f^{-1}(V)\right) \approx H_{C}^{1}\left(E_{+}^{3}\right)$ $=0$ and hence $H_{1}\left(\partial f^{-1}(V)\right)=0$. It follows that $\partial f^{-1}(V)$ is an open 2-cell neighborhood of $f^{-1}(y) \cap \partial M$ lying in $W$; since $W$ was arbitrary, this implies that $f^{-1}(y) \cap \partial M$ is cellular in $\partial M$.

Theorem A (Siebenmann). Suppose $M$ and $N$ are m-manifolds, $m \neq 4$, and $f: M \rightarrow N$ is a proper cell-like map sucb that $S(M, N)$ bolds. If $\epsilon: M \rightarrow(0, \infty)$ is 
continuous, there is a bomeomorphism $g: M \rightarrow N$ such that $d(f(x), g(x))<\epsilon(x)$ for all $x \in M$.

3.2 Theorem. Suppose $M$ and $N$ are m-manifolds, $m \neq 4$, and $f: M \rightarrow N$ is a proper cell-like map sucb that $S(M, N)$ bolds. If $C$ is a closed subset of $M$ sucb that $f^{-1} f(C)=C$, then $f \mid C$ can be extended to a proper map $F: M \rightarrow N$ sucb that $F \mid M-C$ is a bomeomorphism onto $N-f(C)$.

Proof. Let $\rho$ and $d$ be metrices for $M$ and $N$, respectively, and let $\epsilon(x)=$ $\rho(x, C)$ for each $x \in M$. Applying Theorem $A$ to $M-C, N-f(C), f \mid M-C$ and $\epsilon \mid M-C$ yields a homeomorphism $g: M-C \rightarrow N-f(C)$ such that $d(f(x), g(x))<$ $\epsilon(x)$ for every $x \in M-C$. Define a function $F$ on $M$ by $F(x)=f(x)$ if $x \in C$, $F(x)=g(x)$ if $x \in M-C$. It is easily seen that $F$ is continuous and, of course, $F(M)=N$ and $F \mid M-C$ is a homeomorphism onto $N-f(C)$.

That $F$ is proper follows directly from a result of Vãisälä [28] in case $\partial M=$ $\varnothing$; if $\partial M \neq \varnothing, F$ can be shown to be proper by applying Väisälä's result to a manifold obtained by adjoining to $M$ a homeomorphic copy of $M$ which intersects $M$ precise ly in $\partial M$ (and extending $F$ to the resulting manifold in a natural way). Alternatively, it is easy to show directly that $F$ is proper if the function $\epsilon(x)$ is modified so as to guarantee not only that $\epsilon\left(x_{i}\right) \rightarrow 0$ if $\left\{x_{i}\right\} \rightarrow x_{0} \in C$, but also that $\epsilon\left(x_{i}\right) \rightarrow 0$ if $\left\{x_{i}\right\}$ has no accumulation point in $M$. (These properties for $\epsilon(x)$ may be realized easily by choosing $\rho$ to be the metric obtained by restricting to $M$ a metric $\rho^{\prime}$ for the one-point compactification $M+\infty$ of $M$, and defining $\epsilon(x)$ to be $\rho(x, C \cup\{\infty\})$. That $M+\infty$ is metrizable follows [14, p. 247] from the fact that $M$ is locally compact and separable.)

3.3 Theorem. Suppose $M$ is an m-manifold, $m \neq 4$, and $f: M \rightarrow Y$ is a proper cell-like map of $M$ onto a metric space $Y$ such that $S(M, \cap)$ bolds. If for each $y \in Y$ there exist a closed neighborbood $C_{y}$ of $f^{-1}(y)$ in $M$ and a proper map $F_{y}$ of $M$ onto an m-manifold such that $\left\{f^{-1} f(x) \mid x \in C_{y}\right\} \subset\left\{F_{y}^{-1} F_{y}(x) \mid x \in M\right\}$, then $Y$ is bomeomorpbic to $M$.

Proof. In view of Theorem $A$, it is only necessary to show that $Y$ is an $m$ manifold.

Suppose $y_{0} \in Y$. By hypothesis, there exist a closed neighborhood $C$ of $f^{-1}\left(y_{0}\right)$ in $M$ and a proper map $F$ of $M$ onto an m-manifold $N$ such that $\left\{f^{-1} f(x) \mid x \in C\right\} \subset\left\{F^{-1} F(x) \mid x \in M\right\}$. Let $U=\left\{x \in C \mid f^{-1} f(x) \cap C 1(M-C)=\varnothing\right\}$. Since $f$ is proper, it easily follows that $U$ is open in $M$ and that $f^{-1} f(U)=U$. Since $C$ is a neighborhood of $f^{-1}(y)$ in $M, f^{-1}(y) \subset U$. Since $f$ is proper, $U$ is open in $M$, and $f^{-1} f(U)=U, f(U)$ is open in $Y$. The condition $\left\{f^{-1} f(x) \mid\right.$ $x \in C\} \subset\left\{F^{-1} F(x) \mid x \in M\right\}$ implies that also $F^{-1} F(U)=U$ and hence $F(U)$ is 
open in $N$. Since $f \mid U$ and $F \mid U$ are both proper maps, it is easy to show that the function $\phi$ defined on $f(U)$ by $\phi(y)=F\left(f^{-1}(y)\right)$ is a homeomorphism of $f(U)$ onto $F(U)$. Hence $f(U)$ is an open neighborhood of $y_{0}$ in $Y$ which is homeomorphic to an open subset of $N$. It follows that $Y$ is an $m$-manifold, and hence $Y \approx$ M.

3.4 Corollary. If $M$ is an m-manifold, $m \neq 4$, and $G$ is an upper semicontinuous cell-like decomposition of $M$ such that $S(M, G)$ bolds and such that for each $\boldsymbol{g} \in \boldsymbol{G}$ there is a closed neighborbood $C$ of $g$ in $M$ such that $G_{C}$ generates a simple decomposition of $M$, then $G$ is a simple decomposition of $M$.

Combining Theorem 3.2 and Corollary 3.4 yields the following result.

3.5 Corollary. If $M$ is an m-manifold, $m \neq 4$, and $G$ is a cell-like upper semicontinuous decomposition of $M$ such that $S(M, G)$ bolds, then $G$ is simple if and only if it is locally simple.

Remark. It was shown by R. H. Bing [9] that if $Y$ is a closed subset of $E^{3}$ (or $S^{3}$ ) such that each component of $Y$ is compact and does not separate $E^{3}$, and $G$ is the collection of all components of $Y$, then there is a monotone upper semicontinuous decomposition $G^{\prime}$ of $E^{3}$ such that $G \subset G^{\prime}$ and $E^{3} / G^{\prime} \approx$ $E^{3}$. Bing asked $\left[9\right.$, p. 364] whether $G^{\prime}$ could be chosen to be a cellular decomposition in case each element of $G$ is cellular in $E^{3}$. Theorem 3.2 implies that a cell-like upper semicontinuous decomposition $G$ of a closed subset of $E^{3}$ can be extended to a cell-like upper semicontinuous decomposition $G^{\prime}$ of $E^{3}$ such that $E^{3} / G^{\prime} \approx E^{3}$ if and only if the trivial extension of $G$ has this property. Hence, in particular, the set of nondegenerate elements of Bing's dogbone decomposition [8] cannot be extended to any cell-like decomposition of $E^{3}$ which yields $E^{3}$ as its decomposition space. Moreover, if $G$ is any cell-like decomposition of $E^{3}$ such that $E^{3} / G \approx E^{3}$, then by Theorem 3.3 there is an element $g_{0}$ of $G$ such that if $C$ is any closed neighborhood of $g_{0}$ in $E^{3}$, then the set of elements of $G$ which intersect $C$ is not a subset of any upper semicontinuous decomposition $G^{\prime}$ of $E^{3}$-not necessarily cell-like or even monotone-such that $E^{3} / G^{\prime}$ is a 3 -manifold.

We also note that if, in Theorem 3.2, $M=N$ and $C$ does not intersect any nondegenerate $f^{-1}(y), y \in M$, then $f \mid C: C \rightarrow M$ is topologically equivalent to the inclusion $i: C \rightarrow M$. Thus Theorem 3.2 provides a generalization of the results of $\$ 5$ of [3]; in particular, the "repairing" of embeddings in the sense of [6] and [12] cannot be carried out within the category of cell-like maps.

3.6 Lemma. If $K$ is a $k$-manifold, $k \neq 4$, and $f: K \rightarrow K$ is a proper cell-like map sucb that $S(K, f)$ bolds, then for each $y \in K$, there is a locally flat $k$-cell 
$D$ in $K$ such that $D$ is a neigbborbood of $f^{-1}(y)$ in $K$ and (1) $D \cap \partial K=\varnothing$ if $f^{-1}(y) \cap \partial K=\varnothing,(2) D \cap \partial K$ is a locally flat $(k-1)$-cell in $\partial D$ if $f^{-1}(y) \cap \partial K$ $\neq \varnothing$.

Proof. We first observe that if $U$ is an open subset of $K$, then $f^{-1}(U), U$ and $f \mid f^{-1}(U)$ satisfy the hypothesis of Theorem $A$ and hence $f^{-1}(U) \approx U$ (cf. . [5, Theorem 4]). Hence if $y \in \operatorname{Int} K, f^{-1}(y)$ has an open neighborhood in $K$ which is homeomorphic to $E^{k}$, and the existence of a locally flat $k$-cell $D$ in Int $K$ such that $f^{-1}(y) \subset$ Int $D$ follows immediately. On the other hand, if $y \in \partial K$, then $f^{-1}(y)$ has an open neighborhood $V$ in $K$ such that $(V, \partial V) \approx\left(E_{+}^{k}, E^{k-1}\right)$ and the existence of the desired $k$-cell $D$ again follows easily.

Haver [16] has proved that if $M$ is an $m$-cell, $m \neq 5$, then every cell-like map $f: \partial M \rightarrow \partial M$ can be extended to a map $F: M \rightarrow M$ such that $F \mid$ Int $M$ is a homeomorphism onto Int $M$. In the next lemma we give a similar result in a more general setting.

3.7 Lemma. Suppose $K$ is a locally flat $k$-cell in the interior of an $m$-cell $M$, and assume that $k \neq 4$ unless $k=m=4$. Then every cell-like map $f: K \rightarrow K$ for which $S(K, f)$ bolds can be extended to a map $F: M \rightarrow M$ sucb that $F \mid M-K$ is a bomeomorphism onto $M-K$.

Proof. Suppose first that $k=m$, and let $S=\partial K$; by Lemma $3.1, f(S)=S$. If $k=5$, then condition $S(K, f)$ implies that $f \mid S$ is a homeomorphism, and the existence of $F$ follows trivially. Hence assume $k \neq 5$. Then since $f \mid S: S \rightarrow S$ is cell-like and $\operatorname{dim} S=k-1 \neq 4$, it follows from the complement to Theorem $A$, given in [26], that there is a map $\Phi: S \times I \rightarrow S \times I$ such that for each $t \in[0,1)$, $\Phi \mid S \times\{t\}$ is a homeomorphism onto $S \times\{t\}$ and for each $x \in S, \Phi(x, 1)=(f(x), 1)$. Let $A=\mathrm{C} 1(M-K)$; since $K$ is locally flat in Int $M, A \approx S \times l$ and we may identify $A$ and $S \times I$, with. $S$ corresponding to $S \times\{1\}$. Then $\Phi: A \rightarrow M, \Phi \mid S=$ $f \mid S$ and $\Phi \mid A-S$ is a homeomorphism onto $A-S$. If $F: M \rightarrow M$ is defined by $F(x)=f(x)$ if $x \in K, F(x)=\Phi(x)$ if $x \in A$, then $F$ has the desired properties.

Suppose now that $k<m$. Since $K$ is locally flat in Int $M$, it may be assumed that $M$ is the standard $m$-ball $B^{m}$ in $E^{m}$ and that $K \subset \operatorname{Int} B^{k}$, where $B^{k}=$ $\left\{\left(x_{1}, \ldots, x_{m}\right) \in B^{m} \mid x_{i}=0\right.$ for $\left.i>k\right\}$.

Let $U=$ Int $B^{m}$ and $V=\operatorname{Int} B^{k}$. It follows from the first case considered that there is a map $F_{1}: V \rightarrow V$ such that $F_{1} \mid K=f$ and $F_{1} \mid V-K$ is a homeomorphism onto $V-K$. Since $(U, V) \approx\left(E^{m}, E^{k}\right)$, it follows from the Addendum to Corollary 4 of [25] that there is a map $F_{2}: U \rightarrow U$ such that $F_{2}(V)=V,\left\{F_{2}^{-1}(y) \mid\right.$ $y \in V\}=\left\{F_{1}^{-1}(x) \mid x \in V\right\}$ and $F_{2} \mid U-K$ is a homeomorphism onto $U-F_{2}(K)$.

Since $F_{1}$ and $F_{2}$ are proper maps [28] and $\left\{F_{1}^{-1}(x) \mid x \in V\right\}=\left\{F_{2}^{-1}(y) \mid y \in V\right\}$, the function $H_{1}$ defined on $V$ by $H_{1}(y)=F_{1}\left(F_{2}^{-1}(y)\right)$, for each $y \in V$, is a homeomorphism of $V$ onto $V$ and the diagram 


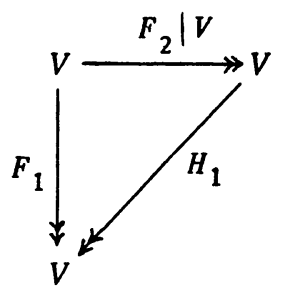

is commutative. It is clear that $H_{1}$ may be extended to a homeomorphism $H_{2}$ : $U \rightarrow U$. If $F^{*}=H_{2} \circ F_{2}$, then $F^{*}: U \rightarrow U, F^{*} \mid K=f$ and $F^{*} \mid U-K$ is a homeomorphism onto $U-K$.

Let $C_{1}$ be a locally flat $m$-cell in $U$ such that $K \subset \operatorname{Int} C_{1}$. Let $S_{1}=\partial C_{1}$ and $S_{2}=F^{*}\left(S_{1}\right)$, and let $C_{2}$ denote the (locally flat) m-cell in $U$ bounded by $\dot{S}_{2} \cdot$. It is easy to verify that $C_{2}=F^{*}\left(C_{1}\right)$ and that $K \subset \operatorname{Int} C_{2}$. Since $K \subset \operatorname{Int} C_{1} \cap$ Int $C_{2}$, there exist homeomorphisms $b_{1}: M \rightarrow C_{1}$ and $b_{2}: M \rightarrow C_{2}$ such that $b_{1} \mid K$ $=b_{2} \mid K=\mathrm{id}_{K}$. If $F=b_{2}^{-1} F^{*} b_{1}$, then $F: M \rightarrow M, F \mid K=f$ and $F \mid M-K$ is a homeomorphism onto $M-K$, as required.

Remark. It is not difficult to show that if $k<m$, the extension $F$ of Lemma 3.7 may be chosen so as to be the identity on $\partial M$; if $k=m$, this is possible if and only if $f \mid \partial K$ is a map of degree 1 . Since an orientation reversing homeomorphism of a closed interval $K$ contained in a half-open interval $M$ cannot be extended homeomorphically to all of $M$, it is clearly impossible to extend Lemma 3.7 directly to the case of an arbitrary locally flat $k$-manifold in the interior of an $m$-manifold. The next theorem shows that such problems can be overcome, in a sense, if one is allowed to change the embedding of $K$ in $M$.

3.8 Theorem. If $K$ is a locally flat k-manifold in the interior of an m-manifold $M, k, m \neq 4$, and $f: K \rightarrow K$ is a proper cell-like map sucb that $S(K, f)$ bolds, then there exist a proper map $F: M \rightarrow M$ and a locally flat embedding $b: K \rightarrow M$ such that the diagram

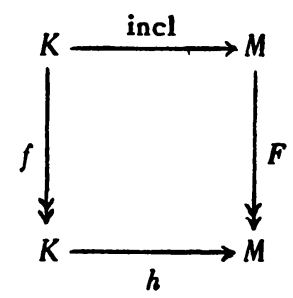

is commutative, and sucb that $F \cdot M-K$ is a bomeomorphism onto $M-b(K)$.

Since the proof of this result seems more naturai in terms of upper semicontinuous decompositions, we give an equivalent formulation of Theorem 3.8 in 
these terms before proceeding $w$ ith the argument.

3.8a Theorem. If $K$ is a locally flat k-manifold in the interior of an $m$ manifold $M, k, m \neq 4$, and $G_{0}$ is a simple decomposition of $K$ sucb that $S\left(K, G_{0}\right)$ bolds, then $G_{0}$ generates a simple decomposition $G$ of $M$, and $K / G_{0}$ is locally flat in $M / G$.

Proof. We will show that each $g \in G$ has a closed neighborhood $C$ in $M$ such that $G_{C}$ generates a simple decomposition of $M$, and it will follow from Corollary 3.4 that $G$ is a simple decomposition of $M$. Clearly we need consider only elements of $G_{0}$.

Suppose $g_{0} \in G_{0}$ and let $D$ be a $k$-cell neighborhood of $g_{0}$ in $K$ satisfying the conditions of Lemma 3.6. Since $G_{0}$ is upper semicontinuous, there exists an open neighborhood $U$ of $g_{0}$ in $K$ such that every element of $G_{0}$ which intersects $\mathrm{Cl} U$ lies in $D-\mathrm{Cl}(K-D)$. Let $G_{1}=\left\{g \in G_{0} \mid g \cap \mathrm{Cl} U \neq \varnothing\right\}$ and let $A$ $=G_{1}^{*}$. Then $A$ is a closed neighborhood of $g_{0}$ in $K, A \subset D-\mathrm{Cl}(K-D)$ and $A$ is saturated with respect to $G_{0}$. Since $g \cap \partial D=g \cap \partial K$ for every $g \in G_{1}$, condition $S\left(D, G_{1}\right)$ holds since $S\left(K, G_{0}\right)$ does.

Let $G_{2}$ denote the decomposition of $D$ generated by $G_{1}$ and $G_{3}$ the decomposition of $K$ generated by $G_{1}$. Let $P_{3}: K \rightarrow K / G_{3}$ be the projection map for $G_{3}$. Since $K / G_{0} \approx K$, it follows from Theorem 3.2 that $K / G_{3} \approx K$. We wish to show that $P_{3}(D) \approx D$, and for this it will be sufficient (applying Theorem $A$ to $\left.D, P_{3}(D), P_{3} \mid D\right)$ to show that $P_{3}(D)$ is a $k$-manifold.

If $x \in D-A$ and $U$ is an open subset of $K$ containing $x$ and lying in $K$ $A$, then since $P_{3} \mid K-A$ is a homeomorphism onto $K-A, P_{3}(U) \cap P_{3}(D) \approx U \cap$ $D$ and hence $P_{3}(x)$ has a neighborhood in $P_{3}(D)$ which is homeomorphic to a $k$ manifold. If $x \in A$, there is an open neighborhood $U$ of $x$ in $K$ such that $U C$ $D-C l(K-D)$ and $U$ is saturated with respect to $G_{3}$. Then $P_{3}(U)$ is open in $P_{3}(K), P_{3}(U) \subset P_{3}(D)$ and, as in the proof of Lemma 3.6, $P_{3}(U) \approx U$. Hence $P_{3}(U)$ is an open subset of $P_{3}(D)$ which contains $P_{3}(x)$ and is homeomorphic to a $k$-manifold. It follows that $P_{3}(D)$ is itself a $k$-manifold, and hence $P_{3}(D)$ $\approx D$. Since $P_{3}(D)=D / G_{2}, G_{2}$ is a simple decomposition of $D$. Since $S\left(D, G_{1}\right)$ holds, it readily follows that $S\left(D, G_{2}\right)$ also holds。

There is a locally flat $m$-cell $B$ in $M$ such that $D \subset$ Int $B$, and there is a closed neighborhood $C$ of $g_{0}$ in $M$ such that $C \subset \underset{\sim}{\operatorname{Int} B}$ and $C \cap K=A$. By Lemma $3.7, G_{2}$ generates a simple decomposition $\tilde{G}_{2}$ of $B$, and $D / G_{2}$. is locally flat in $B / \tilde{G}_{2}$. Since every nondegenerate element of $G$ which intersects $C$ is an element of $G_{1}$ and therefore of $G_{2}$, the decomposition of $B$ generated by $G_{C}$ is identical with that generated by $G_{2}$; hence $G_{C}$ generates a simple decomposition of $B$, and it easily follows that $G_{C}$ generates a simple decomposition $\widetilde{G}_{C}$ of $M$, and that if $\widetilde{P}: M \rightarrow M / \widetilde{G}_{C}$ is the projection map, then $\widetilde{P}(K)$ is locally 
flat in $\tilde{P}(M)$ at the point $\tilde{P}\left(g_{0}\right)$. It follows from Corollary 3.4 that $G$ is a simple decomposition of $M$, and from the observation above that if $P: M \rightarrow M / G$ is the projection map, then $P(K)$ is locally flat in $P(M)$.

Remark. Suppose $K$ is a closed subset of a metric space $M$ and $f$ is a proper map of $K$ onto $K$. If $F_{1}, F_{2}$ are proper maps of $M$ onto $M$ and $b_{1}, b_{2}$ are embeddings of $K$ into $M$ such that, for $i=1,2$, the diagram

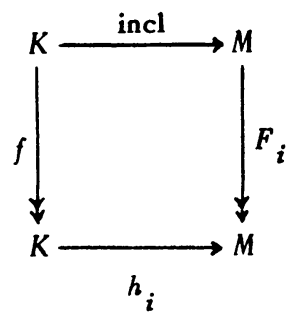

is commutative and $F_{i} \mid M-K$ is a homeomorphism onto $M-b_{i}(K)$, then $\left(F_{1}, b_{1}\right)$ and $\left(F_{2}, b_{2}\right)$ are topologically equivalent, in the sense that there is a homeomorphism $\psi: M \rightarrow M$ such that $\psi \circ F_{1}=F_{2}$ and $\psi \circ b_{1}=b_{2}$. To see this, let $G_{0}=\left\{f^{-1}(x) \mid x \in K\right\}$ and let $G=\tilde{G}_{0}(M)$. Then $\left\{F_{i}^{-1}(x) \mid x \in M\right\}=G, i=1,2$. Let $P: M \rightarrow M / G$ be the projection map and define $\phi_{i}: M / G \rightarrow M$ by $\phi_{i}(y)=$ $F_{i}\left(p^{-1}(y)\right)$. Since $F_{i}$ is a proper map, $\phi_{i}$ is a homeomorphism. It may be verified directly that the homeomorphism $\psi=\phi_{2}{ }^{\circ} \phi_{1}^{-1}: M \rightarrow M$ has the desired properties. It follows, in particular, that if $K, M$ and $f$ satisfy the hypothesis of Theorem 3.8, then for every pair $\left(F^{\prime}, b^{\prime}\right)$ satisfying the above conditions, $b^{\prime}$ is necessarily a locally flat embedding.

With a suitable definition of local flatness, Theorem 3.8 can be shown to hold without the requirement that $K$ be a subset of Int $M$. We sketch below a proof for the case $K \cap \partial M=\partial K$.

3.9 Theorem. In Theorems 3.8 and 3.8a, the bypothesis that $K \subset$ Int $M$ may be replaced by the condition $K \cap \partial M=\partial K$.

Outline of proof. The argument closely parallels that for Theorem 3.8a. We need to show that if $g_{0} \in G_{0}$ and $g_{0} \cap \partial M \neq \varnothing$, then there is a closed neighborhood $C$ of $g_{0}$ in $M$ such that $G_{C}$ generates a simple decomposition $\tilde{G}_{C}$ of $M$, and if $\tilde{P}: M \rightarrow M / \tilde{G}_{C}$ is the projection map, then $\tilde{P}(K)$ is locally flat in $\tilde{P}(M)$ at the point $\widetilde{P}\left(g_{0}\right)$.

As before, we use Lemma 3.6 to obtain a $k$-cell neighborhood $D$ of $g_{0}$ in $K$ such that $D$ is locally flat in $M$ and $D \cap \partial K$ is a locally flat $(k-1)$-cell in $\partial D$. Since $D \cap \partial M=D \cap \partial K$ and $K$ is locally flat in $M$, it follows that $D \cap \partial M$ is locally flat in $\partial M$. We again choose a closed neighborhood $A$ of $g_{0}$ in $K$ such that $A$ is saturated with respect to $G_{0}$ and $A \subset D-\operatorname{Cl}(K-D)$. There 
is a locally flat m-cell $B$ in $M$ such that $D \subset B-\mathrm{Cl}(M-B), B \cap \partial M$ is an $(m-1)$-cell which is locally flat in $M$ and in $\partial B$, and $D \cap \partial M C \operatorname{Int}(B \cap \partial M)$. There is a closed neighborhood $C$ of $g_{0}$ in $M$ such that $C \cap K=A$ and $C \subset B$ We note that $\widetilde{G}_{C}(B)=\left\{g \in G_{0} \mid g \subset A\right\} \cup\{\{p\} \mid p \in B-A\}$, where $\tilde{G}_{C}(B)$ is defined to be the decomposition of $B$ generated by $G_{C}$. In order to prove that $\tilde{G}_{C}(M)$ is a simple decomposition of $M$, it is sufficient to show that $\widetilde{P}(B) \approx B$, where $\widetilde{P}: M \rightarrow M / \tilde{G}_{C}(M)$ is the projection map. We must also, of course, show that $\tilde{P}(D)$ is locally flat in $\tilde{P}(B)$.

As usual, we let $B^{j}$ denote the standard $j$-ball in $E^{j}$. We may identify $B$ with $B^{k-1} \times I \times B^{m-k}$. We let $\mathcal{O}$ denote the center of $B^{m-k}$, and identify $B^{k}$ with $B^{k-1} \times I \times\{0\}$. In view of the local flatness conditions imposed on $D$ and $D \cap \partial M$, we may assume that $D \subset B^{k}$ and $D \cap \partial M \subset \operatorname{Int}\left(B^{k-1} \times\{0\} \times\{O\}\right)$.

The argument used in the proof of The orem 3.8a to show that $P_{3}(D) \approx D$ may be used here to show that $\widetilde{P}\left(B^{k}\right) \approx B^{k}$. Let $\phi: \widetilde{P}\left(B^{k}\right) \rightarrow B^{k}$ be a homeomorphism and let $f=\phi \circ\left(\tilde{P} \mid B^{k}\right)$. Then $f$ is a cell-like map of $B^{k}$ onto $B^{k}$ and $S\left(B^{k}, f\right)$ holds.

Let $L=\partial B^{k}-\operatorname{Int}\left(B^{k-1} \times\{0\} \times\{\mathcal{O}\}\right)$ and define $\epsilon: B^{k} \rightarrow[0, \infty)$ by $G(x)=$ $d(x, L)$. We may apply the complement to Theorem $A$ of $[26]$ to $B^{k}-L, f \mid B^{k}-$ $L$ and $\epsilon \mid B^{k}-L$ to obtain a homotopy $b:\left(B^{k}-L\right) \times I \rightarrow B^{k}-L$ such that $b_{0}$ $=f \mid B^{k}-L$, and for each $t \in(0,1], b_{t}$ is a homeomorphism of $B^{k}-L$ onto itself such that $d\left(b_{t}(x), f(x)\right)<\epsilon(x)$ for all $x \in B^{k}-L$. Define a function $H$ on $B^{k} \times I$ by $H(x, t)=b(x, t)$ if $x \in B^{k}-L, H(x, t)=f(x)$ if $x \in L$. It is easily shown that $H: B^{k} \times I \rightarrow B^{k}, H_{0}=f$, and for each $t \in(0,1], H_{t}$ is a homeomorphism of $B^{k}$ onto $B^{k}$ such that $d(H(x), f(x)) \leq \epsilon(x)$ for all $x \in B^{k}$.

Now define $F$ on $B=B^{k-1} \times I \times B^{m-k}$ by $F(x, s, y)=\left(H_{t}(x, s), y\right)$, where $t$ is the distance from $y$ to $\mathcal{O}$ in $B^{m-k}$. Then $F: B^{m} \rightarrow B^{m}$ and $\left\{F^{-1}(p) \mid p \in B\right\}=\widetilde{G}_{C}(B)$. It follows that $B / \widetilde{G}_{C}(B) \approx F(B)=B$. Since $F\left(B^{k}\right)=$ $B^{k}$ and $F \mid B-B_{\sim}^{k}$ is a homeomorphism onto $B-B^{k}, \tilde{P}\left(B^{k}\right)$ is locally flat in $\widetilde{P}(B)$ and hence $\widetilde{P}(K)$ is locally flat in $\widetilde{P}(M)$ at the point $\widetilde{P}\left(g_{0}\right)$.

As in the proof of Theorem 3.8a, it now follows that $G_{0}$ generates a simple decomposition $G$ of $M$, and $K / G_{0}$ is locally flat in $K / G$.

4. Specializations to $E^{3}$. If $M=E^{3}$ (or $S^{3}$ ), then for every cell-like map $f$ of $M$ onto a metric space $Y$ and for every collection $G$ of cell-like subsets of $M$, conditions $S(M, f)$ and $S(M, G)$ are satisfied. The theorems of $\$ 3$ therefore have somew hat simpler hypotheses if $M=E^{3}$ and we give here a few additional results for this special case.

We will say that a closed subset $Y$ of a metric space $X$ is strongly simply embedded in $X$ if every cell-like upper semicontinuous decomposition of $Y$, simple or not, generates a simple decomposition of $X$. 
The following is an immediate consequence of the Moore theorem [22, Theorem 22] and Theorem 8 of [15]. As usual, we regard $E^{2}$ as the $x y$-plane in $E^{3}$.

4.1 Lemma. Every closed subset of $E^{2}$ is strongly simply embedded in $E^{3}$.

4.2 Theorem. Suppose $X$ is a closed subset of $E^{3}$ and $G$ is a cell-like upper semicontinuous decomposition of $X$. If for each $g \in G$ there is a bomeomorphism of $E^{3}$ onto itself which takes some closed neighborbood of $g$ in $X$ onto a subset of $E^{2}$, then $G$ generates a simple decomposition of $E^{3}$.

Proof. By hypothesis, for each $g \in G$ there is a tame 2-cell $D$ in $E^{3}$ which contains a neighborhood of $g$ in $X$. If $A$ is a closed neighborhood of $g$ in $X$ such that $A \subset D$ and $A$ is saturated with respect to $G$, then by Lemma 4.1, $G_{A}$ generates a simple decomposition of $E^{3}$. Hence, as in the proof of Theorem 3.8a, there is a closed neighborhood $C$ of $g$ in $E^{3}$ such that $G_{C}$ generates a simple decomposition of $E^{3}$, and it follows from Corollary 3.4 that $G$ generates a simple decomposition of $E^{3}$.

4.3 Corollary. Every tame 2-manifold in $E^{3}$ is strongly simply embedded in $E^{3}$.

4.4 Corollary. Every tame 1-dimensional polybedron in $E^{3}$ is strongly simply embedded in $E^{3}$.

Specializing Theorem 3.8a to $E^{3}$ yields the following result (cf. [23, Theorem 4]).

4.5 Theorem. Every tame 3-manifold in $E^{3}$ is simply embedded in $E^{3}$.

5. Examples. Since every proper subarc of an arc $A$ generates a simple decomposition of $A$, it follows immediately that if $A$ is simply embedded in $E^{3}$, then every proper subarc of $A$ must be cellular in $E^{3}$. The arc $A$ itself, however, need not be cellular. To see this, let $A$ be an arc which is wild at each endpoint and locally tame elsewhere. Then $A$ is noncellular [27, Theorem 10], but each proper subarc of $A$ has at most one wild point and is therefore cellular [24, Theorem 3]. If $G$ is a simple decomposition of $A$, then $\widetilde{G}$ has only a countable number of nondegenerate elements, each is a cellular arc and all but at most two are tame. It follows from an easy modification of Theorem 3 of [7] that $E^{3} / \widetilde{G} \approx$ $E^{3}$, and hence $A$ is simply embedded in $E^{3}$.

Similarly, a simple closed curve in $E^{3}$ is simply embedded if it has at most one wild point, but if has two isolated wild points, then it contains a noncellular arc and therefore is not simply embedded. It is possible, however, for 
an everywhere wild simple closed curve (or arc) in $E^{3}$ to be simply embedded.

5.1 Example. There exists a (strongly) simply embedded simple closed curve in $E^{3}$ which is wild at each of its points.

Proof. Let $K$ denote a wild simple closed curve as described by Bothe [11] which has the property that each homeomorphism of $K$ onto $K$ can be extended to a homeomorphism of $E^{3}$ onto $E^{3}$. The curve $K$ is obtained as the intersection of a sequence $\left\{T_{i}\right\}$ of solid tori, with $T_{i+1} \subset$ Int $T_{i}$ for every $i$; each $T_{i}$ is the union of a circular chain $C_{i}=\left\{C_{i 1}, C_{i 2}, \ldots, C_{i n_{i}}\right\}$ of polyhedral 3 -cells of diameter less than $1 / i$ such that the intersection of each two adjacent elements of $\mathcal{C}_{i}$ is a disk which contains exactly one point of $K$, and for each $i$, $\mathcal{C}_{i+1}$ is a refinement of $\mathcal{C}_{i}$. For each $i$, let $F_{i}=K \cap \bigcup_{j=1}^{n_{i}} \partial C_{i j}$ and let $F=$ $\bigcup_{i=1}^{\infty} F_{i}$. Then $F$ is a countable dense subset of $K$, and for each $i, F_{i}$ is finite and $F_{i+1} \subset F_{i}$.

Suppose $G$ is a cell-like upper semicontinuous decomposition of $K$. Let $H$ denote the set of nondegenerate elements of $G$ and let $D$ be a countable dense subset of $K-H^{*}$. Since $F$ and $F \cup D$ are countable dense subsets of the simple closed curve $K$, there is a homeomorphism $\phi: K \rightarrow K$ such that $\phi(F \cup D)=F$. Let $F^{\prime}=\phi(D)$ and let $H_{1}=\phi(H)$.

Suppose $U$ is an open subset of $E^{3}$ containing $H_{1}^{*}$ and let $A$ be an element of $H_{1}$. Since $F^{\prime}$ is a dense subset of $K-H_{1}^{*}$, each endpoint of $A$ is a limit point of $F^{\prime}$ and it follows that there is an arc $A^{\prime} \subset K \cap U$ such that $A \subset$ Int $A^{\prime}$ and the endpoints of $A^{\prime}$ belong to $F^{\prime}$. Since each element of $\mathcal{C}_{i}$ has diameter less than $1 / i$, there is an integer $n$ such that if $i>n$, every element of $\mathcal{C}_{i}$ which intersects $A^{\prime}$ lies in $U$. Since $F_{i} \supset F_{i+1}$ for every $i$, there is an integer $i_{0}>n$ such that both endpoints of $A^{\prime}$ belong to $F_{i}$. If $C$ denotes the union of all the elements of $\mathcal{C}_{i_{0}}$ whose interiors intersect $A^{q}$, then $C$ is a tame 3-cell lying in $U, A \subset$ Int $C$, and $K \cap \partial C=\partial A^{\prime} \subset F^{\prime}$. Since $H_{1}^{*} \cap F^{\prime}=\varnothing$, repetition of this process yields a null family of disjoint tame 3-cells in $U$ such that each element of $H_{1}$ lies in the interior of some member of this family, and hence by Theorem 3 of [1], $H_{1}$ generates a simple decomposition of $E^{3}$.

Since $K$ is "homogeneously embedded," $\phi$ can be extended to a homeomorphism $\Phi: E^{3} \rightarrow E^{3}$. Then $\Phi(\tilde{G})$ is the decomposition of $E^{3}$ generated by $\Phi(H)$. Since $\Phi(H)=\phi(H)=H_{1}$ and $H_{1}$ generates a simple decomposition of $E^{3}$, so does $H$. Hence $G$ generates a simple decomposition of $E^{3}$, and it follows that $K$ is strongly simply embedded in $E^{3}$.

We note that there exist nondegenerate continua in $E^{3}$ for which every embedding into $E^{3}$ is a simple embedding. Such are, for example, the continua described by Whyburn [29] and Cook [13] which have no simple decomposition other than the trivial decomposition into singletons. The next example shows 
that the other extreme is also possible-a continuum may be embeddable in $E^{3}$, yet have no simple embedding at all.

We first collect some facts about upper semicontinuous decompositions of metric.spaces. Let $X$ be a metric space and $G$ a collection of pairwise disjoint compact subsets of $X$ such that $G^{*}=X$. Then it is known that $G$ is an upper semicontinuous decomposition of $X$ if and only if the following condition holds: for each sequence $\left\{p_{i}\right\}, p_{i} \in g_{i} \in G$, which converges to a point of $g \in G$ and any sequence $\left\{q_{i}\right\}, q_{i} \in g_{i}$, some subsequence of $\left\{q_{i}\right\}$ converges to a point of $g$.

The following observation, which is an easy consequence of the above characterization of upper semicontinuous decompositions, will be useful: If $G$ is an upper semicontinuous decomposition of a metric space $X$ and $f$ is a proper map of $X$ onto a metric space $Y$, then $f(G)$ is an upper semicontinuous decomposition of $Y$ provided the elements of $f(G)$ are disjoint. (Note that it is not required that $f$ map distinct elements of $G$ onto distinct elements of $f(G)$, but only that if $g, g^{\prime} \in G$ and $f(g) \cap f\left(g^{\prime}\right) \neq \varnothing$, then $f(g)=f\left(g^{\prime}\right)$.)

The next lemma may be compared with Theorem 20 of [19].

5.2 Lemma. If $X$ is a compact metric space, $G$ and $G_{1}$ are upper semicontinuous decompositions of $X$ such that $G_{1}$ is a refinement of $G$, and $P_{1}$ is the projection map of $X$ onto $X / G_{1}$, then $P_{1}(G)$ is an upper semicontinuous decomposition of $X / G_{1}$ and $X / G \approx\left(X / G_{1}\right) / P_{1}(G)$.

Proof. That $P_{1}(G)$ is upper semicont inuous follows immediately from the observation made above.

To complete the proof, let $X_{1}=X / G_{1}, G_{2}=P_{1}(G)$ and $Y=X_{1} / G_{2}$. Let $P_{2}$ : $X_{1} \rightarrow Y$ be the projection map associated with $G_{2}$ and let $f=p_{2} \circ p_{1}$. Then $f:$ $X \rightarrow Y$, and in order to show that $Y \approx X / G$ it is sufficient to show that for each $g \in G, f^{-1}(f(g))=g$. Since $f^{-1} f(g)=P_{1}^{-1} P_{2}^{-1} P_{2} P_{1}(g)=P_{1}^{-1}\left(P_{2}^{-1} P_{2}(g)\right)$, where $g_{2}=P_{1}(g)$, and $P_{2}^{-1} P_{2}\left(g_{2}\right)=g_{2}$ (because $g_{2} \in G_{2}$ and $P_{2}$ is the projection map for $\left.G_{2}\right)$, it follows that $f^{-1}(f(g))=P_{1}^{-1}\left(P_{1}(g)\right)$.

If $x \in P_{1}^{-1}\left(P_{1}(g)\right)$, then $P_{1}(x) \in P_{1}(g)$ and hence there is an $x^{\prime} \in g$ such that $P_{1}(x)=P_{1}\left(x^{\prime}\right)$. Let $g_{1}$ be the element of $G_{1}$ containing $x$ and $g_{1}^{\prime}$ the element of $G_{1}$ containing $x^{\prime}$. Then $g_{1}^{\prime}=P_{1}^{-1}\left(P_{1}\left(x^{\prime}\right)\right)=P_{1}^{-1}\left(P_{1}(x)\right)=g_{1}$. Since $x^{\prime} \epsilon$ $g \cap g_{1}^{\prime}$ and $G_{1}$ is a refinement of $G, g_{1}^{\prime} \subset g$. Since $x \in g_{1}=g_{1}^{\prime} \subset g, x \in g$. Hence $P_{1}^{-1}\left(P_{1}(g)\right) \subset g$ and therefore $P_{1}^{-1}\left(P_{1}(g)\right)=g$.

5.3 Example. There exists a compact absolute retract in $E^{3}$ which bas no simple embedding in $E^{3}$.

Proof. Let $B_{0}$ denote the standard unit ball in $E^{3}$ and let $B_{1}, B_{2}, \ldots$ be a sequence of spherical balls, each with center on the $x$-axis, such that, for $i=1,2, \ldots, B_{i-1}$ and $B_{i}$ are externally tangent at a point $p_{i}$, and such that 
the sequence $\left\{B_{i}\right\}_{i=0}^{\infty}$ converges to a point $p$. For each $i$ let $B_{-i}$ denote the reflection of $B_{i}$ in the $y z-$ plane, and let $p_{-i}$ be the reflection of $p_{i}$. Let $M$ denote the closure of the union of the balls $B_{i}, i=0, \pm 1, \pm 2, \ldots$.

Let $A_{0}$ be an arc from $p_{-1}$ to $p_{1}$ which lies except for its endpoints in Int $B_{0}$ and which is wild at $p_{1}$ and locally tame at all other points. For $i=$ $1,2, \ldots$, let $\phi_{i}$ be a homeomorphism of $B_{0}$ onto $B_{i}$ which takes $p_{-1}$ onto $p_{i}$ and $p_{1}$ onto $p_{i+1}$, and let $A_{i}=\phi\left(A_{0}\right)$. Let $A=\bigcup_{i=2}^{\infty} A_{i} \cup\{p\}$ and let $X=$ $M / A$ (i.e., $X$ is the decomposition space of $M$ given by the decomposition whose only nondegenerate element is $A$ ).

Since $A$ is cellular in $E^{3}$ [24, Theorem 3$], E^{3} / A \approx E^{3}$ and hence since $X=$ $M / A=E^{3} / A, X$ is embeddable in $E^{3}$. Since $M$ and $A$ are absolute retracts, so is $X[10, \mathrm{p} .131]$. It remains to be shown that $X$ has no simple embedding in $E^{3}$; this will be done by showing that $X$ contains an arc $K$ such that $X / K \approx X$ and such that if $f: X \rightarrow E^{3}$ is any embedding, then $f(K)$ is not cellular in $E^{3}$. It will follow that $f(X) / f(K) \approx f(X)$ but $E^{3} / f(K)$ \&o $E^{3}$, and therefore $f(X)$ is not simply embedded in $E^{3}$.

Let $P: M \rightarrow M / A$ be the projection map. There is a homeomorphism of $M$ onto itself which takes $A_{0} \cup A_{1} \cup A$ onto $A$, and hence $M /\left(A_{0} \cup A_{1} \cup A\right) \approx$ $M / A=X$. By Lemma 5.2, $M /\left(A_{0} \cup A_{1} \cup A\right) \approx(M / A) / P\left(A_{0} \cup A_{1}\right)$, and it follows that $X \approx X / P\left(A_{0} \cup A_{1}\right)$.

Let $A_{0}^{-}$denote the reflection of $A_{0}$ in the $y z$-plane and let $K=P\left(A_{0}^{-} \cup A_{1}\right)$. Let $C=\mathrm{Cl}\left(M-B_{0}\right)$ and let $B_{0}^{\prime}$ and $C^{\prime}$ be disjoint copies of $B_{0}$ and $C$, respectively. Let $A_{0}^{\prime}$ be the arc in $B_{0}^{\prime}$ corresponding to $A_{0}$, and let $A_{1}^{\prime}$ and $A^{\prime}$ be the arcs in $C^{\prime}$ corresponding to $A_{1}$ and $A$, respectively. Let $p_{-1}^{\prime}, p_{1}^{\prime}$ be the points of $B_{0}^{\prime}$ corresponding to $p_{-1}$ and $p_{1}$, and let $p_{-1}^{N}, p_{1}^{n}$ be the points of $C^{\prime}$ corresponding to $p_{-1}$ and $p_{1}$. Let $M^{\prime}=B_{0}^{\prime} \cup C^{\prime}$, and let $f$ be a map of $M^{\prime}$ onto $M$ which takes $\left\{p_{-1}^{n}, p_{-1}^{\prime}\right\}$ onto $p_{-1}$, and $\left\{p_{1}^{\prime}, p_{1}^{N}\right\}$ onto $p_{1}$, such that $f$ is $1-1$ on $M^{\prime}-\left\{p_{-1}^{\prime \prime}, p_{-1}^{\prime}, p_{1}^{\prime}, p_{1}^{\prime \prime}\right\}$ and takes $A_{0}^{\prime} \cup A_{1}^{\prime} \cup A^{\prime}$ onto $A_{0} \cup A_{1} \cup A$. Let $g$ be a map of $M^{\prime}$ onto $M$ which takes $\left\{p_{-1}^{\prime \prime}, p_{1}^{\prime}\right\}$ onto $p_{-1}$, and $\left\{p_{-1}^{\prime}, p_{1}^{\prime \prime}\right\}$ onto $p_{1}$, such that $g$ is $1-1$ on $M^{\prime}-\left\{p_{-1}^{N}, p_{-1}^{\prime}, p_{1}^{\prime}, p_{1}^{\prime \prime}\right\}$ and takes $A_{0}^{\prime} \cup A_{1}^{\prime} \cup A^{\prime}$ onto $A_{0}^{-} \cup A_{1} \cup A$. Then by Lemma 5.2, $M^{\prime} /\left(A_{0}^{\prime} \cup A_{1}^{\prime} \cup A^{\prime}\right) \approx f\left(M^{\prime}\right) / f\left(A_{0}^{\prime} \cup A_{1}^{\prime} \cup A^{\prime}\right)=$ $M /\left(A_{0} \cup A_{1} \cup A\right) \approx X$ and $M^{\prime} /\left(A_{0}^{\prime} \cup A_{1}^{\prime} \cup A^{\prime}\right) \approx g(M) / g\left(A_{0}^{\prime} \cup A_{1}^{\prime} \cup A^{\prime}\right)=$ $M /\left(A_{0}^{-} \cup A_{1} \cup A\right)$. It follows that $X \approx M /\left(A_{0}^{-} \cup A_{1} \cup A\right) \approx(M / A) / P\left(A_{0}^{-} \cup A_{1}\right)=$ $X / K$.

If $f: X-E^{3}$ is an embedding and $g=f \mid P\left(B_{0} \cup B_{1}\right)$, then $g$ is an embedding of the union of the 3-cells $P\left(B_{0}\right)$ and $P\left(B_{1}\right)$ and it follows [21, Lemma 4] that $f(K)$ is wild at each of its endpoints. Since $f(K)$ is locally tame at all other points except possibly $f\left(P\left(p_{1}\right)\right), f(K)$ is not cellular in $E^{3}$ [27, Theorem 10] and it follows, as indicated above, that $f(X)$ is not simply embedded in $E^{3}$. 
6. Questions and further comments. In order that a closed set $X \subseteq E^{n}$ should be simply embedded, it is necessary that each element of any simple decomposition of $X$ be cellular in $E^{n}(n \neq 4)$. That this condition is not sufficient in general may be seen from the following example: Let $H$ denote the set of nondegenerate elements of Bing's dogbone decomposition [8], constructed so that each element of $H$ has its upper endpoint on a given horizontal plane $\alpha$ and otherwise lies wholly below $a$, and let $X$ denote the union of $H^{*}$ and all vertical intervals of length 1 with lower endpoint in $a \cap H^{*}$. Then if $G$ is any monotone decomposition of $X$, each element of $G$ is either a point or a tame arc; $X$ is not simply embedded in $E^{3}$, however, since the decomposition of $X$ whose nondegenerate elements are the elements of $H$ is a simple decomposition of $X$ which does not generate a simple decomposition of $E^{3}$. Whether the condition that each element of each simple decomposition of $X$ be cellular in $E^{n}$ is sufficient, in certain special cases, to insure that $X$ be simply embedded is the gist of our first question.

6.1 Suppose $X$ is a closed subset of $E^{n}$. Which, if any, of the following conditions implies that $X$ is simply embedded in $E^{n}$ ?

(a) $X$ is an arc and every proper subarc of $X$ is cellular in $E^{n}$.

(b) $X$ is a simple closed curve and every arc in $X$ is cellular in $E^{n}$.

(c) $X$ is a $k$-manifold and every $k$-cell in $X$ is cellular in $E^{n}, k<n$.

(d) $X$ is an n-manifold and every $(n-1)$-cell in $\partial X$ is cellular in $E^{n}$.

It was remarked in $\$ 4$ that every 1 -dimensional polyhedron in $E^{3}$ is strongly simply embedded. Not every 2 -dimensional polyhedron in $E^{3}$ is strongly simply embedded, however, since, for example, if $X$ is a "book-with-one-page" (the product of a triod and an interval), then $X$ contains a noncellular arc and hence has a cell-like decomposition which does not generate a simple decomposition of $E^{3}$. In this case, at least, it appears that every simple decomposition of $X$ generates a simple decomposition of $E^{3}$, which suggests the following question.

6.2 Is every polybedron in $E^{3}$ simply embedded in $E^{3}$ ?

Example 5.3 is a (3-dimensional) absolute retract in $E^{3}$ which has no simple embedding in $E^{3}$, and it seems likely that a 2-dimensional AR with this property could be obtained similarly by replacing each $B_{i}$ of Example 5.3 by a book-withone-page. Every 1-dimensional AR, however, is embeddable in $E^{2}$ and hence, by Lemma 4.1, has a (strongly) simple embedding in $E^{3}$; dropping the "absolute retract" condition leads to the following question (which we conjecture has a negative answer).

6.3 Does every 1-dimensional continuum bave a simple embedding in $E^{3}$ ?

\section{REFERENCES}

1. Steve Armentrout, Upper semi-continuous decompositions of $E^{3}$ with at most countably many non-degenerate elements, Ann. of Math (2) 78 (1963), 605-618. MR 27 \#6255. 
2. Steve Armentrout, Concerning cellular decompositions of 3-manifolds that yield 3-manifolds, Trans. Amer. Math. Soc. 133 (1968), 307-332. MR 37 \#5859.

3. - Concerning cellular decompositions of 3-manifolds with boundary, Trans. Amer. Math. Soc. 137 (1969), 231-236. MR 38 \#5224.

4. Cellular decompositions of 3-manifolds that yield 3-manifolds, Bull.

Amer. Math. Soc. 75 (1969), 453-456. MR 39 \#9 35.

5. $\longrightarrow$ Cellular decompositions of 3-manifolds that yield 3-manifolds, Mem Amer. Math. Soc. No. 107 (1970).

6. Ralph J. Bean, Repairing embeddings and decompositions in $S^{3}$, Duke Math. J. 36 (1969), 379-385. MR 39 \#4820.

7. R. H. Bing, Upper semicontinuous decompositions of $E^{3}$, Ann. of Math. (2) 65 (1957), 363-374. MR 19, 1187.

8. A decomposition of $E^{3}$ into points and tame arcs such that the decomposition space is topologically different from $E^{3}$, Ann. of Math. (2) 65 (1957), 484-500. MR 19,1187.

9. - Extending monotone decompositions of 3-manifolds, Trans. Amer. Math. Soc. 149 (1970), 351-369. MR $41 \# 7656$.

10. Karol Borsuk, Theory of retracts, Monografie Mat., Tom 44, PWN, Warsaw, 1967. MR $35 \# 306$.

11. H. G. Bothe, Ein homogen wilder Knoten, Fund. Math. 60 (1967), 271-283. MR $35 \# 327$.

12. W. S. Boyd, Repairing embeddings of 3-cells with monotone maps of $E^{3}$, Trans. Amer. Math. Soc. 161 (1971), 123-144. MR 43 \#864.

13. H. Cook, Continua which admit only the identity mapping onto non-degenerate subcontinua, Fund. Math. 60 (1967), 241-249. MR 36 \#3315.

14. James Dugundji, Topology, Allyn and Bacon, Boston, Mass., 1966. MR 33 \#1824.

15. E. Dyer and M. E. Hamstrom, Completely regular mappings, Fund. Math. 45 (1958), 103-118. MR 19, 1187.

16. William Haver, A characterization theorem for cellular maps, Bull, Amer. Math. Soc. 76 (1970), 1277-1280. MR 42 \#2440.

17. R. C. Lacher, Cell-like mappings, I, Pacific J. Math. 30 (1969), 717-731. MR $40 \# 4941$.

18. H. W. Lambert, Some comments on the structure of compact decompositions of $S^{3}$, Proc. Amer. Math. Soc. 19 (1968), 180-184. MR 37 \#901.

19. Jack W. Lamoreaux, Decompositions of metric spaces with a 0 -dimensional set of non-degenerate elements, Canad. J. Math. 21 (1969), 202-216. MR 39 \#2122.

20. Louis F. McAuley, Some fundamental theorems and problems related to monotone mappings, Proc. First Conf. on Monotone Mappings and Open Mappings (SUNY at Bingham ton, Binghamton, N.Y., 1970), State Univ. of New York at Binghamton, Binghamton, NY., 1971, pp. 1-36. MR $44 \# 4722$.

21. D. R. McMillan, Some topological properties of piercing points, Pacific J. Math. 22 (1967), 313-322. MR $35 \$ 7319$.

22. R. L. Moore, Concerning upper semicontinuous collections of continua, Trans. Amer. Math. Soc. 27 (1925), 416-428.

23. V. Nicholson, Mapping cylinder neighborhoods, Trans. Amer. Math. Soc. 143 (1969), 259-268. MR $40 \# 2038$. 
24. R. B. Sher, Determining the cellularity of a 1-complex by properties of its ares, Proc. Amero Math. Soc. 26 (1970), 491-498. MR 42 \#5242.

25. - Realizing cell-like maps in Euclidean space, General Topology and Appl. 2 (1972), 75-89.

26. L. C. Siebenmann, Approximating cellular maps by homeomorphisms, Topology 11 (1972), 271-294. MR $45 \# 4431$.

27. C. D. Sikkema, A duality between certain spheres and arcs in $S^{3}$, Trans. Amer. Math. Soc. 122 (1966), 399-415. MR $33 \$ 7991$.

28. J. Vãisälä, Invariance of domain under acyclic mappings, Duke Math. J. 33 (1966), 679-681. MR 34 \#815.

29. G. T. Whyburn, A continum every subcontinuum of which separates the plane, Amer. J. Math. 52 (1930), 319-330.

30. - Analytic topology, Amer. Math. Soc. Colloq. Publ., vol. 28, Amer. Math. Soc., Providence, R.I., 1942. MR 4, 86.

DEPARTMENT OF MATHEMATICS, UNIVERSITY OF GEORGIA, ATHENS, GEORGIA 30602 